\title{
Nocardimicins G, H and I, Siderophores with Muscarinic M3 Receptor Binding Inhibitory Activity from Nocardia nova JCM 6044
}

\author{
Yoshitaka Ikeda, Tamotsu Furumai, Yasuhiro Igarashi
}

Received: June 20, 2005 / Accepted: August 29, 2005

(C) Japan Antibiotics Research Association

\begin{abstract}
In the screening for muscarinic M3 receptor binding inhibitors from microbial secondary metabolites, the extract of Nocardia nova JCM 6044 was found to be highly active. Bioassay-guided isolation led to the identification of three siderophores, nocardimicins G (1), H (2) and I (3). Their chemical structures were determined by spectroscopic analysis using NMR and MS. 1 and 2 inhibited the binding of tritium-labeled $\mathrm{N}$-methylscopolamine to the muscarinic M3 receptor with $K_{\mathrm{i}}$ values of $0.44 \mu \mathrm{M}$ and $0.37 \mu \mathrm{M}$, respectively, whereas 3 showed no inhibition at $10 \mu \mathrm{M}$. 1 and $\mathbf{2}$ also showed weak binding inhibitory activity to the M5 receptor but not to the $\mathrm{M} 1, \mathrm{M} 2$ and $\mathrm{M} 4$ receptors at $10 \mu \mathrm{M}$.
\end{abstract}

Keywords Nocardia nova JCM 6044, siderophore, muscarinic M3 receptor, binding assay

\section{Introduction}

Parasympatholytics block the acetylcholine-mediated transmission of impulses from postganglionic parasympathetic fibers to the organ of response by competitive antagonism at muscarinic receptors. Due to the resulting relaxing effect on smooth muscles, these substances are also called neurotropic spasmolytic agents. To date, five muscarinic acetylcholine receptor genes, $m 1 \sim m 5$, have been cloned [1]. Although four subtypes of muscarinic receptors $(\mathrm{M} 1 \sim \mathrm{M} 4)$ have been pharmacologically defined, pharmacological characterization of the M5 receptor has not yet been accomplished. All the subtypes known to date belong to the superfamily of membrane-bound receptors coupled to G-proteins [2].

Muscarinic receptors are widely distributed in the central and peripheral nervous systems as well as in the parasympathetically innervated organs of response. All five subtypes occur in the central nervous system. The smooth muscle of the respiratory tract, gastrointestinal tract, and urinary bladder contains a heterogeneous population of M2 and M3 receptors. As contraction of smooth muscle generally reflected M3 pharmacology alone [3], the M3 receptor may represent a therapeutic target for the treatment of respiratory disorders such as chronic obstructive pulmonary disease, gastrointestinal disorders such as irritable bowel syndrome, and urinary tract disorders such as urinary incontinence [4].

We have previously reported the isolation of cremastrine, an alkaloid from Cremastra appendiculata (Orchidaceae) [5] and nocardimicins A F, siderophores from Nocardia sp. TP-A0674 [6], as selective muscarinic M3 receptor binding inhibitors. In our ongoing search for the muscarinic M3 inhibitors from natural products, three siderophores, nocardimicins G (1), H (2) and I (3) were identified in the culture broth of Nocardia nova JCM 6044. This paper describes the isolation, structure determination, and biological activity of $\mathbf{1} \sim \mathbf{3}$.
Y. Ikeda (Corresponding author): Pharmaceuticals Research Unit, Mitsubishi Pharma Corporation, 1000, Kamoshida-cho, Aoba-ku, Yokohama, Kanagawa 227-0033, Japan, E-mail: ikeda.yoshitaka@mc.m-pharma.co.jp
T. Furumai, Y. Igarashi: Biotechnology Research Center, Toyama Prefectural University, 5180, Kurokawa, Kosugi, Toyama 939-0398, Japan 


\section{Materials and Methods}

\section{Microorganism}

The producing microorganism, strain Nocardia nova JCM 6044, was purchased from Japanese Collection of Microorganisms. It was maintained on an ISP medium No. 2 slant at $10^{\circ} \mathrm{C}$ for laboratory use.

\section{Instrumental Analysis}

Melting points were determined on a Yanaco micro melting point apparatus. Optical rotations were measured on a Jasco P-1020 digital polarimeter. NMR spectra were measured on a Bruker AMX-500 NMR spectrometer using standard Bruker pulse programs. Chemical shifts are given in $\delta$ values with reference to tetramethylsilane as an internal standard. IR spectra were recorded on a Perkin Elmer 1725X FT-TR spectrophotometer. LC-MS spectra were measured on an Agilent MSD spectrometer and highresolution LC-MS spectra were measured on a JEOL JMS700 spectrometer. The LC-MS/MS spectra were measured on a Finnigan TSQ QUANTUM Ultra spectrometer (Thremo electron, MA). UV spectra were recorded on a Hitachi U-3010 spectrophotometer.

\section{Biological Assay}

The binding affinities $\left(K_{\mathrm{i}}\right)$ to five receptor subtypes were determined according to the method previously described [5], by inhibition of specific binding of tritium labeled $N$ methylscopolamine $\left(\left[{ }^{3} \mathrm{H}\right]-\mathrm{NMS}\right)$ using membranes from insect Sf9 cells expressing human $m 1 \sim m 5$ receptors at MDS Pharma Services (Taiwan).

\section{Results and Discussion}

\section{Fermentation}

A loopful of a mature slant culture of strain Nocardia nova JCM 6044 was inoculated into five 500-ml K-1 flasks containing $100 \mathrm{ml}$ of the seed medium consisting of soluble starch $1 \%$, glucose $0.5 \%$, NZ-case $0.3 \%$, yeast extract $0.2 \%$, tryptone $0.5 \%, \mathrm{~K}_{2} \mathrm{HPO}_{4} 0.1 \%$, and $\mathrm{CaCO}_{3} 0.3 \%(\mathrm{pH}$ 7.0). The flask was incubated at $32^{\circ} \mathrm{C}$ for 5 days on a rotary shaker $(200 \mathrm{rpm})$. Three-ml aliquots of the seed culture were transferred into fifty $500-\mathrm{ml} \mathrm{K}-1$ flasks each containing $100 \mathrm{ml}$ of the production medium consisting of glycerol $4 \%$, NZ-case $0.5 \%$, Pharmamedia $2 \%, \mathrm{CaCO}_{3}$ $0.5 \%$ and HP-20 $1 \%$. The $\mathrm{pH}$ of the medium was adjusted to 7.0 before sterilization. Fermentation was carried out at $32^{\circ} \mathrm{C}$ for 5 days on a rotary shaker $(200 \mathrm{rpm})$.

\section{Isolation}

The fermented whole broth (5 liters) was centrifuged $(5,000 \mathrm{rpm}, 10$ minutes $)$ to separate the mycelium. The supernatant was discarded and the mycelium was extracted by agitating in acetone (4 liters). The mycelium was removed by centrifugation and acetone was removed by evaporation. The resultant aqueous solution was extracted with EtOAc $(300 \mathrm{ml} \times 5)$ and the EtOAc layer was evaporated to dryness in vacuo. The EtOAc extract (3.06 g) was fractionated into three active fractions (fraction $\mathrm{A} \sim \mathrm{C}$ ) by preparative HPLC using an ODS column (Delta-Pak C18, $40 \mathrm{~mm}$ i.d. $\times 200 \mathrm{~mm}$, Waters, MA) with a linear $\mathrm{CH}_{3} \mathrm{CN}-0.2 \%$ HOAc (aq) gradient from 50 to $100 \%$ $\mathrm{CH}_{3} \mathrm{CN}$ over 20 minutes at a flow rate of $80 \mathrm{ml} /$ minute. The fraction A $(120.6 \mathrm{mg})$ was subjected to preparative HPLC using an ODS column (XTerra Prep RP18 C18, 19 mm i.d. $\times 150 \mathrm{~mm}$, Waters, MA) with the eluent of $\mathrm{CH}_{3} \mathrm{CN}-10 \mathrm{mM}$ $\mathrm{NH}_{4} \mathrm{OAc}$ (aq) (pH 9.0, isocratic elution, 57:43) at a flow rate of $20 \mathrm{ml} /$ minute. Active fractions were combined and evaporated in vacuo to give $\mathbf{1}(83.1 \mathrm{mg})$ as an active principle. Similarly, $2(67.0 \mathrm{mg})$ and $3(13.8 \mathrm{mg})$ were isolated from the fractions B $(333.8 \mathrm{mg})$ and C $(96.6 \mathrm{mg})$, respectively.

\section{Structure Determination}

Physico-chemical properties of nocardimicins G (1), H (2) and I (3) are summarized in Table 1. Compound $\mathbf{2}$ was obtained as pale yellow amorphous solid. The molecular formula of 2 was established as $\mathrm{C}_{42} \mathrm{H}_{67} \mathrm{O}_{10} \mathrm{~N}_{5}$ on the basis of the HR-LC/MS and ${ }^{13} \mathrm{C}$ NMR data. The ${ }^{1} \mathrm{H}$ NMR spectrum (Table 2) of $\mathbf{2}$ revealed the presence of two amide protons $(\delta 8.68$ and 8.00$)$, a 1,2-disubstituted benzene moiety $(\delta 7.68,7.32,7.05$ and 6.82$)$ and two methyl groups ( $\delta 1.35$ and $\delta 0.87$ ). In the ${ }^{13} \mathrm{C}$ NMR and DEPT spectra of 2 , signals assignable to 42 carbons were detected, including four carbonyl carbons $(\delta 172.6,172.3,170.8$ and 169.4), six aromatic carbons $(\delta 160.6,134.2,129.0,119.2,117.3$ and 111.2), one quaternary $s p^{2}$ carbon adjacent to a heteroatom $(\delta 167.5)$, one oxymethine carbon $(\delta 77.1)$, one nitrogen bearing methylene carbon ( $\delta 52.8)$, and two nitrogen bearing methine carbons ( $\delta 53.7$ and 52.0). Due to the existence of cis and trans conformations around the formamide bond, the NMR signals for some protons and carbons were observed in pairs at $27^{\circ} \mathrm{C}$. These pairs of NMR signals were merged into one peak at $67^{\circ} \mathrm{C}$ except for the $N$-formyl and $\varepsilon$-methylene group. The remaining carbon signals were assigned to two methyl, 22 methylene, two methine carbons, and one quaternary carbon (Table 3 ).

2D-NMR analysis of 2 allowed the identification of four partial structures $\mathrm{A} \sim \mathrm{D}$ : a hydroxyphenyloxazoline moiety, $N_{\varepsilon}$-formyllysine, 2-methyl-3-hydroxy hexadecanoate and $\mathcal{\varepsilon}$ - 
Table 1 Physico-chemical properties of nocardimicins G (1), H (2) and I (3)

\begin{tabular}{|c|c|c|c|}
\hline & 1 & 2 & 3 \\
\hline Appearance & Pale yellow amorphous & Pale yellow amorphous & Pale yellow amorphous \\
\hline MP & $108 \sim 112^{\circ} \mathrm{C}$ & $152 \sim 155^{\circ} \mathrm{C}$ & $132 \sim 136^{\circ} \mathrm{C}$ \\
\hline$[\alpha]_{D}^{25}$ & $-3.9(c 1.0, \mathrm{MeOH})$ & $-5.8(c 1.0, \mathrm{MeOH})$ & $-9.5(c 1.0, \mathrm{MeOH})$ \\
\hline \multicolumn{4}{|l|}{ HR-LC-MS } \\
\hline Found: & $774.4649[\mathrm{M}+\mathrm{H}]^{+}$ & $802.4933[\mathrm{M}+\mathrm{H}]^{+}$ & $830.5243[\mathrm{M}+\mathrm{H}]^{+}$ \\
\hline Calcd: & 774.4653 (for $\mathrm{C}_{40} \mathrm{H}_{64} \mathrm{O}_{10} \mathrm{~N}_{5}$ ) & 802.4966 (for $\mathrm{C}_{42} \mathrm{H}_{68} \mathrm{O}_{10} \mathrm{~N}_{5}$ ) & $830.5279\left(\right.$ for $\mathrm{C}_{44} \mathrm{H}_{72} \mathrm{O}_{10} \mathrm{~N}_{5}$ ) \\
\hline Molecular formula & $\mathrm{C}_{40} \mathrm{H}_{63} \mathrm{O}_{10} \mathrm{~N}_{5}$ & $\mathrm{C}_{42} \mathrm{H}_{67} \mathrm{O}_{10} \mathrm{~N}_{5}$ & $\mathrm{C}_{44} \mathrm{H}_{71} \mathrm{O}_{10} \mathrm{~N}_{5}$ \\
\hline$U V \lambda_{\max } \mathrm{nm}(\log \varepsilon)$ & 243 (4.02), 249 (4.03), 305 (3.64) & 243 (4.03), 249 (4.04), 305 (3.66) & $243(4.02), 249(4.03), 305$ (3.65) \\
\hline \multirow[t]{2}{*}{$\mathrm{IR} v_{\max }\left(\mathrm{cm}^{-1}\right)$} & $2925,2855,2360,1731$ & $2924,2854,2360,1732$ & $2923,2853,2360,1730$ \\
\hline & $1645,1538,1463,1368$ & $1645,1538,1466,1368$ & $1645,1538,1470,1367$ \\
\hline
\end{tabular}

aminocaprolactam (Fig. 2). Connectivities of these partial structures were elucidated by the HMBC experiment. Important HMBC correlations are indicated in Fig. 2. The connectivity between the partial structures $\mathrm{A}$ and $\mathrm{B}$ was confirmed by the long-range coupling from $\mathrm{H}-13$ to $\mathrm{C}-12$. The HMBC correlation from $\mathrm{H}-17$ to $\mathrm{C}-15$ established the linkage between partial structures $\mathrm{B}$ and $\mathrm{C}$ through an ester bond and that from $\mathrm{H}-20$ to $\mathrm{C}-19$ revealed the linkage of the partial structures $\mathrm{C}$ and $\mathrm{D}$ through an amide bond. In consideration of the molecular formula, the remaining two hydroxyl groups were assigned as substituents at the nitrogen atoms $\mathrm{N}-23$ and $\mathrm{N}-32$. The positive-mode LC-MS/MS spectrum of $\mathbf{2}$ indicated that fragment ions corresponding to mycobactic acid [7] moiety and cobactine moiety at $\mathrm{m} / \mathrm{z} 380$ and 441, respectively (Fig. 3). Based on these spectral data, the structure of nocardimicin H (2) was established as shown (Fig. 1).

The molecular formulae of nocardimicin G (1) and I (3) were established as $\mathrm{C}_{40} \mathrm{H}_{63} \mathrm{O}_{10} \mathrm{~N}_{5}$ and $\mathrm{C}_{44} \mathrm{H}_{71} \mathrm{O}_{10} \mathrm{~N}_{5}$, respectively, on the basis of the HR-LC-MS and ${ }^{13} \mathrm{C}$ NMR data. These compounds showed ${ }^{1} \mathrm{H}$ and ${ }^{13} \mathrm{C}$ NMR spectra (Table 2 and 3) quite similar to those of nocardimicin $\mathrm{H}$ (2). In the LC-MS/MS spectra, the fragment ion derived from the mycobactic acid moiety was detected at $\mathrm{m} / \mathrm{z} 380$ as in the case of 2, while the fragment mass derived from the cobactin moiety of 1 was 28 mass units smaller $(\mathrm{m} / z$ 413) and that of 3 was 28 mass units larger $(m / z 469)$. These observations led to the confirmation that $\mathbf{1}$ and $\mathbf{3}$ are the congeners of $\mathbf{2}$ differing in the fatty acid chain length (Fig. 3).

1 possesses a planar structure identical with BMS199687 [8] and A-77543 [9]. BMS-199687 has been isolated from Actinomadura ferruginea and shown to have in vitro and in vivo antitumor activity. A-77543 is a metabolite of a Nocardia and shows antitumor activity in vivo and anti-HIV activity. Although these three compounds show similar NMR spectra and optical rotations $\left\{1:[\alpha]_{\mathrm{D}}^{22}-6.9^{\circ}\left(c 1.0, \mathrm{CHCl}_{3}\right)\right.$ and $[\alpha]_{\mathrm{D}}^{25}-3.9^{\circ}$ (c 1.0, MeOH), BMS-199687: $[\alpha]_{\mathrm{D}}^{25}-11.4^{\circ}\left(c 1.0, \mathrm{CHCl}_{3}\right)$, A-77543: $\left.\left.[\alpha]_{\mathrm{D}}^{20}-7.1^{\circ}(c) 1.0, \mathrm{MeOH}\right)\right\}$, it is uncertain whether they are the same compound. The planar structure of $\mathbf{2}$ is identical with that of brasilibactin A, a cytotoxic compound isolated from Nocardia brasilieneis [10]. The optical rotations of these compounds showed significant differences $\left\{2\right.$ : $[\alpha]_{\mathrm{D}}^{22}-10.7^{\circ}\left(c 1.0, \mathrm{CHCl}_{3}\right)$, brasilibactin $\left.\mathrm{A}:[\alpha]_{\mathrm{D}}^{22}-31^{\circ}\left(c 1.0, \mathrm{CHCl}_{3}\right)\right\}$, suggesting that they are stereoisomers. Elucidation of the stereochemistry of $\mathbf{1} \sim \mathbf{3}$ is under investigation.

\section{Biological Properties}

The inhibitory activities of $\mathbf{1} \sim \mathbf{3}$ to muscarinic M1 M5 receptor binding are summarized in Table 4. 1 and 2 inhibited the binding of $\left[{ }^{3} \mathrm{H}\right]-\mathrm{NMS}$ to the muscarinic M3 receptor with a $K_{\mathrm{i}}$ value of $0.44 \mu \mathrm{M}$ and $0.37 \mu \mathrm{M}$, respectively, while 3 showed no inhibition. This finding suggest that the fatty acid chain moiety play a role in the interaction of these compounds with the muscarinic M3 receptor like nocardimicins $\mathrm{A} \sim \mathrm{F}[6]$. They also showed inhibitory activity to M5 receptor binding but not to binding to the M1, M2 and M4 receptors at $10 \mu \mathrm{M}$ (Table 4). It is noteworthy that nocardimicins exhibit high subtype-selectivity against the M3 receptor. Currently used parasympatholytic drugs can be chemically divided into two classes: compounds derived from tropane alkaloids and synthetic quaternary ammonium compounds with diverse chemical structures. None of these substances possesses the desirable property of sufficient subtype-selective blockage of M3 receptors that mediate contraction. The largely nonselective blockage of all muscarinic receptor subtypes explains some of the side effects such as increase in the 
Table $2{ }^{1} \mathrm{H}$ NMR Data $(\delta)$ for $\mathbf{1} \sim \mathbf{3}$ in pyridine- $d_{5}\left(67^{\circ} \mathrm{C}\right)$

\begin{tabular}{|c|c|c|c|}
\hline Position & 1 & 2 & 3 \\
\hline 3 & $7.05(1 \mathrm{H}$, br.d, $J=8.3 \mathrm{~Hz})$ & $7.05(1 \mathrm{H}$, br.d, $J=8.3 \mathrm{~Hz})$ & $7.05(1 \mathrm{H}$, br.d, $J=8.4 \mathrm{~Hz})$ \\
\hline 4 & $7.32(1 \mathrm{H}, \mathrm{dt}, J=7.8,1.7 \mathrm{~Hz})$ & $7.32(1 \mathrm{H}$, br.t, $J=7.8 \mathrm{~Hz})$ & $7.32(1 \mathrm{H}$, br.t, $J=7.1 \mathrm{~Hz})$ \\
\hline 5 & $6.82(1 \mathrm{H}$, br.t, $J=7.6 \mathrm{~Hz})$ & $6.82(1 \mathrm{H}$, br.t, $J=7.6 \mathrm{~Hz})$ & $6.81(1 \mathrm{H}$, br.t, $J=7.5 \mathrm{~Hz})$ \\
\hline 6 & $7.68(1 \mathrm{H}, \mathrm{dd}, J=7.8,1.8 \mathrm{~Hz})$ & $7.68(1 \mathrm{H}, \mathrm{dd}, J=7.8,1.1 \mathrm{~Hz})$ & $7.68(1 \mathrm{H}, \mathrm{dd}, J=7.8,1.2 \mathrm{~Hz})$ \\
\hline \multirow[t]{2}{*}{9} & $4.88(1 \mathrm{H}, \mathrm{m})^{\mathrm{a})}$ & $4.89(1 \mathrm{H}, \mathrm{m})^{\mathrm{a})}$ & $4.89(1 \mathrm{H}, \mathrm{m})^{\mathrm{a})}$ \\
\hline & $4.52(1 \mathrm{H}, \mathrm{dd}, J=10.2,8.5 \mathrm{~Hz})$ & $4.53(1 \mathrm{H}, \mathrm{dd}, J=10.1,8.7 \mathrm{~Hz})$ & $4.53(1 \mathrm{H}, \mathrm{dd}, J=10.1,8.7 \mathrm{~Hz})$ \\
\hline 10 & $5.17(1 \mathrm{H}, \mathrm{dd}, J=10.2,7.7 \mathrm{~Hz})$ & $5.17(1 \mathrm{H}, \mathrm{dd}, J=10.2,7.8 \mathrm{~Hz})$ & $5.18(1 \mathrm{H}, \mathrm{dd}, J=10.2,7.8 \mathrm{~Hz})$ \\
\hline $13(\mathrm{NH})$ & $8.70(1 \mathrm{H}, \mathrm{d}, J=7.3 \mathrm{~Hz})$ & $8.68(1 \mathrm{H}, \mathrm{d}, J=7.2 \mathrm{~Hz})$ & $8.79(1 \mathrm{H}, \mathrm{d}, J=6.5 \mathrm{~Hz})$ \\
\hline 14 & $4.94(1 \mathrm{H}$, br.dd, $J=13.6,7.9 \mathrm{~Hz})$ & $4.94(1 \mathrm{H}, \mathrm{br} . \mathrm{dd}, J=13.7,8.0 \mathrm{~Hz})$ & $4.94(1 \mathrm{H}, \mathrm{br} . \mathrm{dd}, J=13.7,8.0 \mathrm{~Hz})$ \\
\hline 17 & $5.47(1 \mathrm{H}, \mathrm{dt}, J=12.3,6.1 \mathrm{~Hz})$ & $5.47(1 \mathrm{H}, \mathrm{dt}, J=12.3,6.1 \mathrm{~Hz})$ & $5.49(1 \mathrm{H}, \mathrm{dt}, J=12.3,6.1 \mathrm{~Hz})$ \\
\hline 18 & $2.92(1 \mathrm{H}, \mathrm{dq}, J=6.8,6.5 \mathrm{~Hz})$ & $2.92(1 \mathrm{H}, \mathrm{dq}, J=6.8,6.5 \mathrm{~Hz})$ & $2.92(1 \mathrm{H}, \mathrm{dq}, J=6.8,6.5 \mathrm{~Hz})$ \\
\hline $20(\mathrm{NH})$ & $8.01(1 \mathrm{H}, \mathrm{d}, J=6.6 \mathrm{~Hz})$ & $8.00(1 \mathrm{H}, \mathrm{d}, J=6.3 \mathrm{~Hz})$ & $8.07(1 \mathrm{H}, \mathrm{d}, J=6.5 \mathrm{~Hz})$ \\
\hline 21 & $4.88(1 \mathrm{H}, \mathrm{m})^{\mathrm{a})}$ & $4.89(1 \mathrm{H}, \mathrm{m})^{\mathrm{a})}$ & $4.89(1 \mathrm{H}, \mathrm{m})^{\mathrm{a})}$ \\
\hline 24 & $3.82 \sim 3.72(2 \mathrm{H}, \mathrm{m})$ & $3.82 \sim 3.72(2 \mathrm{H}, \mathrm{m})$ & $3.82 \sim 3.72(2 \mathrm{H}, \mathrm{m})$ \\
\hline 25 & $1.60 \sim 1.50(2 \mathrm{H}, \mathrm{m})^{\mathrm{b})}$ & $1.60 \sim 1.50(2 \mathrm{H}, \mathrm{m})^{\mathrm{b})}$ & $1.60 \sim 1.50(2 \mathrm{H}, \mathrm{m})^{\mathrm{b})}$ \\
\hline \multirow[t]{2}{*}{26} & $1.80 \sim 1.70(1 \mathrm{H}, \mathrm{m})^{\mathrm{c})}$ & $1.80 \sim 1.70(1 \mathrm{H}, \mathrm{m})^{\mathrm{c})}$ & $1.80 \sim 1.70(1 \mathrm{H}, \mathrm{m})^{\mathrm{cl}}$ \\
\hline & $1.70 \sim 1.60(1 \mathrm{H}, \mathrm{m})^{\mathrm{d})}$ & $1.70 \sim 1.60(1 \mathrm{H}, \mathrm{m})^{\mathrm{d})}$ & $1.70 \sim 1.60(1 \mathrm{H}, \mathrm{m})^{\mathrm{d})}$ \\
\hline \multirow[t]{2}{*}{27} & $2.16 \sim 2.10(1 \mathrm{H}, \mathrm{m})^{\mathrm{e})}$ & $2.16 \sim 2.10(1 \mathrm{H}, \mathrm{m})^{\mathrm{e})}$ & $2.16 \sim 2.10(1 \mathrm{H}, \mathrm{m})^{\mathrm{e})}$ \\
\hline & $1.60 \sim 1.50(1 \mathrm{H}, \mathrm{m})^{\mathrm{b})}$ & $1.60 \sim 1.50(1 \mathrm{H}, \mathrm{m})^{\mathrm{b})}$ & $1.60 \sim 1.50(1 \mathrm{H}, \mathrm{m})^{\mathrm{b})}$ \\
\hline \multirow[t]{2}{*}{28} & $2.16 \sim 2.10(1 \mathrm{H}, \mathrm{m})^{\mathrm{e})}$ & $2.16 \sim 2.10(1 \mathrm{H}, \mathrm{m})^{\mathrm{e})}$ & $2.16 \sim 2.10(1 \mathrm{H}, \mathrm{m})^{\mathrm{e})}$ \\
\hline & $2.02 \sim 1.91(1 \mathrm{H}, \mathrm{m})$ & $2.02 \sim 1.91(1 \mathrm{H}, \mathrm{m})$ & $2.02 \sim 1.91(1 \mathrm{H}, \mathrm{m})$ \\
\hline 29 & $1.70 \sim 1.60(2 \mathrm{H}, \mathrm{m})^{\mathrm{d})}$ & $1.70 \sim 1.60(2 \mathrm{H}, \mathrm{m})^{\mathrm{d})}$ & $1.70 \sim 1.60(2 \mathrm{H}, \mathrm{m})^{\mathrm{d})}$ \\
\hline 30 & $1.80 \sim 1.70(2 \mathrm{H}, \mathrm{m})^{\mathrm{c})}$ & $1.80 \sim 1.70(2 \mathrm{H}, \mathrm{m})^{\mathrm{c})}$ & $1.80 \sim 1.70(2 \mathrm{H}, \mathrm{m})^{\mathrm{cl}}$ \\
\hline 31 & $3.70 \sim 3.50(2 \mathrm{H}$, br.s $)$ & $3.70 \sim 3.50(2 \mathrm{H}$, br.s $)$ & $3.70 \sim 3.50(2 \mathrm{H}$, br.s $)$ \\
\hline \multirow[t]{2}{*}{33} & $8.79(0.6 \mathrm{H}, \mathrm{br} . \mathrm{s})^{f)}$ & $8.79(0.6 \mathrm{H}, \mathrm{br} . \mathrm{s})^{\mathrm{f})}$ & $8.79(0.6 \mathrm{H}, \mathrm{br} . \mathrm{s})^{\mathrm{f})}$ \\
\hline & $8.18(0.4 \mathrm{H} \text {, br.s })^{f)}$ & $8.18(0.4 \mathrm{H} \text {, br.s })^{f)}$ & $8.18(0.4 \mathrm{H} \text {, br.s })^{f)}$ \\
\hline 34 & $1.87 \sim 1.80(2 \mathrm{H}, \mathrm{m})$ & $1.87 \sim 1.80(2 \mathrm{H}, \mathrm{m})$ & $1.87 \sim 1.80(2 \mathrm{H}, \mathrm{m})$ \\
\hline 35 & $1.60 \sim 1.50(2 \mathrm{H}, \mathrm{m})^{\mathrm{b})}$ & $1.60 \sim 1.50(2 \mathrm{H}, \mathrm{m})^{\mathrm{b})}$ & $1.60 \sim 1.50(2 \mathrm{H}, \mathrm{m})^{\mathrm{b})}$ \\
\hline 36 & $1.32 \sim 1.20(2 \mathrm{H}, \mathrm{m})^{\mathrm{g})}$ & $1.32 \sim 1.20(2 \mathrm{H}, \mathrm{m})^{\mathrm{g})}$ & $1.32 \sim 1.20(2 \mathrm{H}, \mathrm{m})^{\mathrm{g})}$ \\
\hline 37 & $1.32 \sim 1.20(2 \mathrm{H}, \mathrm{m})^{\mathrm{g})}$ & $1.32 \sim 1.20(2 \mathrm{H}, \mathrm{m})^{\mathrm{g})}$ & $1.32 \sim 1.20(2 \mathrm{H}, \mathrm{m})^{\mathrm{g})}$ \\
\hline 38 & $1.32 \sim 1.20(2 \mathrm{H}, \mathrm{m})^{\mathrm{g})}$ & $1.32 \sim 1.20(2 \mathrm{H}, \mathrm{m})^{\mathrm{g})}$ & $1.32 \sim 1.20(2 \mathrm{H}, \mathrm{m})^{\mathrm{g})}$ \\
\hline 39 & $1.32 \sim 1.20(2 \mathrm{H}, \mathrm{m})^{\mathrm{g})}$ & $1.32 \sim 1.20(2 \mathrm{H}, \mathrm{m})^{\mathrm{g})}$ & $1.32 \sim 1.20(2 \mathrm{H}, \mathrm{m})^{\mathrm{g})}$ \\
\hline 40 & $1.32 \sim 1.20(2 \mathrm{H}, \mathrm{m})^{\mathrm{g})}$ & $1.32 \sim 1.20(2 \mathrm{H}, \mathrm{m})^{\mathrm{g})}$ & $1.32 \sim 1.20(2 \mathrm{H}, \mathrm{m})^{\mathrm{g})}$ \\
\hline 41 & $1.32 \sim 1.20(2 \mathrm{H}, \mathrm{m})^{\mathrm{g})}$ & $1.32 \sim 1.20(2 \mathrm{H}, \mathrm{m})^{\mathrm{g})}$ & $1.32 \sim 1.20(2 \mathrm{H}, \mathrm{m})^{\mathrm{g})}$ \\
\hline 42 & $1.32 \sim 1.20(2 \mathrm{H}, \mathrm{m})^{\mathrm{g})}$ & $1.32 \sim 1.20(2 \mathrm{H}, \mathrm{m})^{\mathrm{g})}$ & $1.32 \sim 1.20(2 \mathrm{H}, \mathrm{m})^{\mathrm{g})}$ \\
\hline 43 & $1.32 \sim 1.20(2 \mathrm{H}, \mathrm{m})^{\mathrm{g})}$ & $1.32 \sim 1.20(2 \mathrm{H}, \mathrm{m})^{\mathrm{g})}$ & $1.32 \sim 1.20(2 \mathrm{H}, \mathrm{m})^{\mathrm{g})}$ \\
\hline 44 & $1.32 \sim 1.20(2 \mathrm{H}, \mathrm{m})^{\mathrm{g})}$ & $1.32 \sim 1.20(2 \mathrm{H}, \mathrm{m})^{\mathrm{g})}$ & $1.32 \sim 1.20(2 \mathrm{H}, \mathrm{m})^{\mathrm{g})}$ \\
\hline 45 & $1.40 \sim 1.30(2 \mathrm{H}, \mathrm{m})$ & $1.32 \sim 1.20(2 \mathrm{H}, \mathrm{m})^{\mathrm{g})}$ & $1.32 \sim 1.20(2 \mathrm{H}, \mathrm{m})^{\mathrm{g})}$ \\
\hline 46 & $0.87(3 \mathrm{H}$, br.t, $J=6.9 \mathrm{~Hz})$ & $1.32 \sim 1.20(2 \mathrm{H}, \mathrm{m})^{\mathrm{g})}$ & $1.32 \sim 1.20(2 \mathrm{H}, \mathrm{m})^{\mathrm{g})}$ \\
\hline 47 & - & $1.40 \sim 1.30(2 \mathrm{H}, \mathrm{m})$ & $1.32 \sim 1.20(2 \mathrm{H}, \mathrm{m})^{\mathrm{g})}$ \\
\hline 48 & - & $0.87(3 \mathrm{H}$, br.t, $J=6.9 \mathrm{~Hz})$ & $1.32 \sim 1.20(2 \mathrm{H}, \mathrm{m})^{\mathrm{g})}$ \\
\hline 49 & - & - & $1.40 \sim 1.30(2 \mathrm{H}, \mathrm{m})$ \\
\hline 50 & - & - & $0.86(3 \mathrm{H}$, br.t, $J=7.0 \mathrm{~Hz})$ \\
\hline 51 & $1.35(3 \mathrm{H}, \mathrm{d}, J=7.0 \mathrm{~Hz})$ & $1.35(3 \mathrm{H}, \mathrm{d}, J=6.9 \mathrm{~Hz})$ & $1.35(3 \mathrm{H}, \mathrm{d}, J=7.0 \mathrm{~Hz})$ \\
\hline
\end{tabular}

a),b),c),d),el,g) Overlapped each other.

f) Observed as broadened signals at $67^{\circ} \mathrm{C}$. Described chemical shifts were recoded at $27^{\circ} \mathrm{C}$. 
Table $3{ }^{13} \mathrm{C}$ NMR data $(\delta)$ for $\mathbf{1} \sim \mathbf{3}$ in pyridine- $d_{5}\left(67^{\circ} \mathrm{C}\right)$

\begin{tabular}{|c|c|c|c|}
\hline Position & 1 & 2 & 3 \\
\hline 1 & 111.2 (s) & 111.2 (s) & 111.1 (s) \\
\hline 2 & 160.6 (s) & 160.6 (s) & 160.6 (s) \\
\hline 3 & $117.3(d)$ & $117.3(d)$ & $117.3(d)$ \\
\hline 4 & 134.2 (d) & 134.2 (d) & $134.2(d)$ \\
\hline 5 & 119.2 (d) & 119.2 (d) & 119.1 (d) \\
\hline 6 & 129.0 (d) & 129.0 (d) & $128.9(d)$ \\
\hline 7 & 167.5 (s) & 167.5 (s) & 167.5 (s) \\
\hline 9 & $69.9(\mathrm{t})$ & $69.9(t)$ & $69.9(\mathrm{t})$ \\
\hline 10 & $68.9(d)$ & 68.9 (d) & $68.9(d)$ \\
\hline 12 & 170.8 (s) & 170.8 (s) & 170.8 (s) \\
\hline 14 & $53.8(d)$ & 53.7 (d) & 53.7 (d) \\
\hline 15 & $173.7(s)^{a)}$ & $172.6(s)^{a)}$ & $172.6(s)^{a)}$ \\
\hline 17 & 77.1 (d) & 77.1 (d) & 77.1 (d) \\
\hline 18 & 45.0 (d) & $45.0(d)$ & $45.0(d)$ \\
\hline 19 & $172.7(\mathrm{~s})^{\mathrm{a})}$ & $172.3(\mathrm{~s})^{\mathrm{a})}$ & $172.3(\mathrm{~s})^{\mathrm{a})}$ \\
\hline 21 & 52.1 (d) & $52.0(d)$ & $52.0(d)$ \\
\hline 22 & 169.4 (s) & 169.4 (s) & 169.4 (s) \\
\hline 24 & $52.8(t)$ & $52.8(t)$ & $52.8(\mathrm{t})$ \\
\hline 25 & $26.5(t)$ & $26.5(t)$ & $26.1(\mathrm{t})$ \\
\hline 26 & $28.0(\mathrm{t})$ & $27.9(\mathrm{t})$ & $27.9(\mathrm{t})$ \\
\hline 27 & $31.7(t)^{b)}$ & $31.7(\mathrm{t})^{\mathrm{b})}$ & $31.7(t)^{b)}$ \\
\hline 28 & $31.9(t)^{b)}$ & $31.9(\mathrm{t})^{\mathrm{b})}$ & $31.9(t)^{b)}$ \\
\hline 29 & $23.4(\mathrm{t})$ & $23.4(t)$ & $23.4(t)$ \\
\hline 30 & $27.2(\mathrm{t})$ & $27.2(t)$ & $27.2(\mathrm{t})$ \\
\hline 31 & $50.0,46.6(t)^{c)}$ & $50.0,46.6(t)^{c)}$ & $50.0,46.6(t)^{c)}$ \\
\hline 33 & $163.0,157.3(d)^{c)}$ & $163.0,157.3(d)^{c)}$ & $163.0,157.3(\mathrm{~d})^{\mathrm{c})}$ \\
\hline 34 & $31.9(t)^{b)}$ & $31.9(t)^{b)}$ & $31.9(t)^{b)}$ \\
\hline 35 & $26.1(\mathrm{t})$ & $26.1(\mathrm{t})$ & $26.1(\mathrm{t})$ \\
\hline 36 & $30.0 \sim 29.6(t)^{d)}$ & $30.0 \sim 29.6(t)^{d)}$ & $30.0 \sim 29.6(\mathrm{t})^{\mathrm{d})}$ \\
\hline 37 & $30.0 \sim 29.6(\mathrm{t})^{\mathrm{d})}$ & $30.0 \sim 29.6(t)^{d)}$ & $30.0 \sim 29.6(\mathrm{t})^{\mathrm{d})}$ \\
\hline 38 & $30.0 \sim 29.6(t)^{d)}$ & $30.0 \sim 29.6(t)^{d)}$ & $30.0 \sim 29.6(\mathrm{t})^{\mathrm{d})}$ \\
\hline 39 & $30.0 \sim 29.6(t)^{d)}$ & $30.0 \sim 29.6(t)^{d)}$ & $30.0 \sim 29.6(\mathrm{t})^{\mathrm{d})}$ \\
\hline 40 & $30.0 \sim 29.6(t)^{d)}$ & $30.0 \sim 29.6(\mathrm{t})^{\mathrm{d})}$ & $30.0 \sim 29.6(t)^{d)}$ \\
\hline 41 & $30.0 \sim 29.6(t)^{d)}$ & $30.0 \sim 29.6(t)^{d)}$ & $30.0 \sim 29.6(\mathrm{t})^{\mathrm{d})}$ \\
\hline 42 & $30.0 \sim 29.6(t)^{d)}$ & $30.0 \sim 29.6(t)^{d)}$ & $30.0 \sim 29.6(\mathrm{t})^{\mathrm{d})}$ \\
\hline 43 & $30.0 \sim 29.6(t)^{d)}$ & $30.0 \sim 29.6(t)^{d)}$ & $30.0 \sim 29.6(\mathrm{t})^{\mathrm{d})}$ \\
\hline 44 & $32.2(t)^{b)}$ & $30.0 \sim 29.6(t)^{d)}$ & $30.0 \sim 29.6(\mathrm{t})^{\mathrm{d})}$ \\
\hline 45 & $22.9(\mathrm{t})$ & $30.0 \sim 29.6(\mathrm{t})^{\mathrm{d})}$ & $30.0 \sim 29.6(t)^{d)}$ \\
\hline 46 & $14.2(q)$ & $32.2\left(t^{b)}\right.$ & $30.0 \sim 29.6(\mathrm{t})^{\mathrm{d})}$ \\
\hline 47 & - & $22.9(t)$ & $30.0 \sim 29.6(\mathrm{t})^{\mathrm{d})}$ \\
\hline 48 & - & 14.2 (q) & $32.1(t)^{b)}$ \\
\hline 49 & - & - & $22.9(\mathrm{t})$ \\
\hline 50 & - & - & $14.1(q)$ \\
\hline 51 & $14.0(q)$ & $13.9(q)$ & $14.0(q)$ \\
\hline
\end{tabular}

a),b) Assignments may be interchangeable.

c) Observed as broadened signals at $67^{\circ} \mathrm{C}$. Described chemical shifts were recoded at $27^{\circ} \mathrm{C}$.

d) Overlapped each other. 


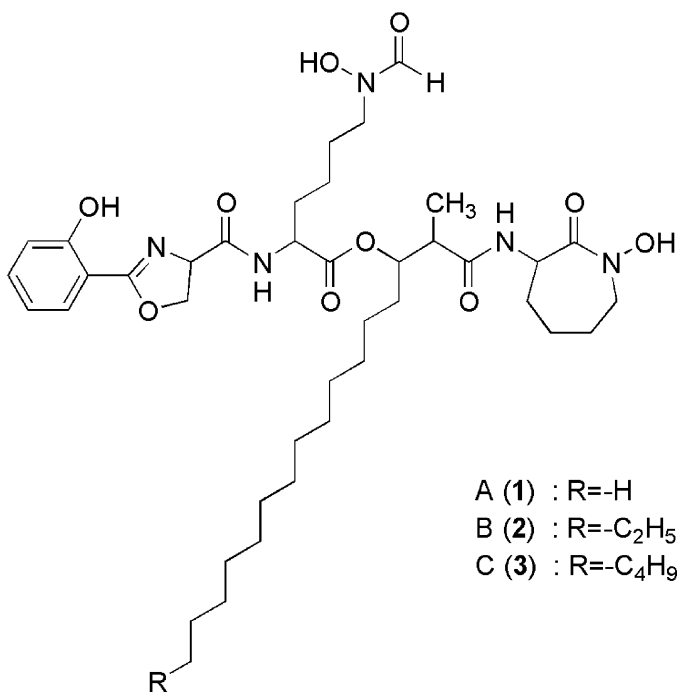

Fig. 1 Structures of nocardimicins A (1), B (2) and C (3).

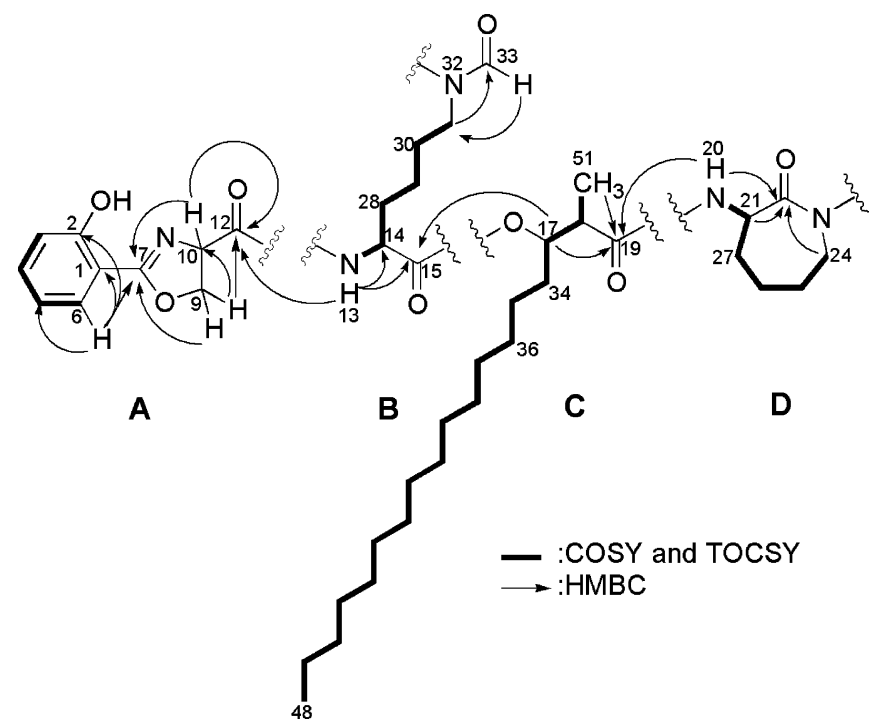

Fig. 2 Key COSY and HMBC correlations for 2.

cardiac rate that might be caused by the blockage of cardiac M2 receptors. For control of smooth muscle spasms, the development of subtype selective antagonists with fewer side effects is necessary. Nocardimicins are the new lead compound for the design of selective M3 antagonists. Further studies on the pharmacological functions of the nocardimicins are in progress.

Acknowledgment We thank Mr. T. Ogawa for HR-LC/MS and LC-MS/MS measurements.

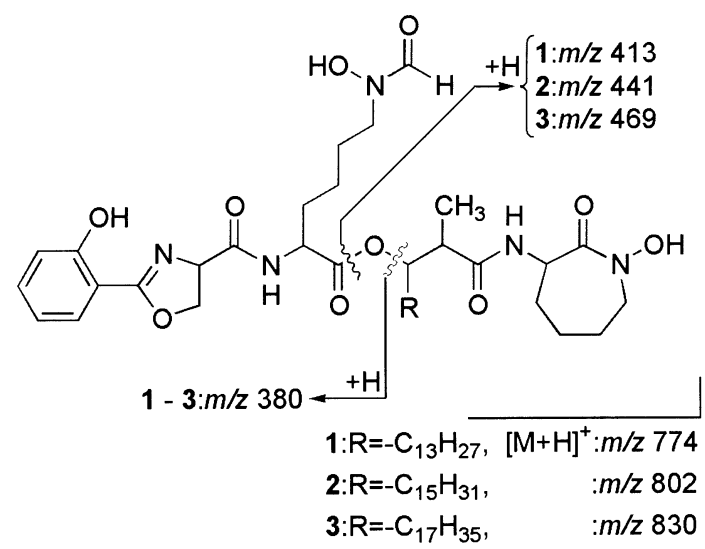

Fig. 3 Fragment ions of $\mathbf{1} \sim \mathbf{3}$ in LC-MS/MS (positive mode).

Table $4 \quad I C_{50}$ values $(\mu \mathrm{M})$ of $\mathbf{1} \sim \mathbf{3}$ to muscarinic M1 M5 receptors

\begin{tabular}{cccc}
\hline Receptor & $\mathbf{1}$ & $\mathbf{2}$ & $\mathbf{3}$ \\
\hline M1 & $>10$ & $>10$ & $>10$ \\
M2 & $>10$ & $>10$ & $>10$ \\
M3 & $2.06(0.44)^{a)}$ & $1.75(0.37)^{\mathrm{a})}$ & $>10$ \\
M4 & $>10$ & $>10$ & $>10$ \\
M5 & $2.07(1.49)^{\text {a) }}$ & $4.00(2.87)^{\text {a) }}$ & $>10$ \\
\hline$K_{\mathrm{i}}$ values $(\mu \mathrm{M})$ are in parentheses. & & \\
& & & \\
& & &
\end{tabular}

\section{References}

1. Kubo T, Fukuda K, Mikami A, Maeda A, Takahashi H, Mishina M, Haga T, Haga K, Ichiyama A, Kanagawa K, Kojima M, Matsuno H, Hirose T, Numa S. Cloning, sequencing and expression of complementary DNA encoding the muscarinic acetylcholine receptor. Nature 323: 411-416 (1986)

2. Cockcroft S, Gomperts GD. Role of guanine nucleotide binding protein in the activation of polyphosphoinositide phosphodiesterase. Nature 314: 534-536 (1985)

3. Eglen RM, Hegde SS, Watson N. Muscarinic receptor subtypes and smooth muscle function. Pharmacol Rev 48: 531-565 (1996)

4. Wallis RM. Pre-clinical and clinical pharmacology of selective muscarinic M3 receptor antagonists. Life Sci 56: 861-868 (1995)

5. Ikeda Y, Nonaka H, Furumai T, Igarashi Y. Cremastrine, a pyrrolozidine alkaloid from Cremastra appendiculata. J Nat Prod 68: 572-573 (2005)

6. Ikeda Y, Nonaka H, Furumai T, Onaka H, Igarashi Y. Nocardimicins A, B, C, D, E, and F, siderophores with muscarinic M3 receptor inhibiting activity from Nocardia 
sp. TP-A0674. J Nat Prod 68: 1061-1065 (2005)

7. Snow G. A. Mycobactions: Iron-Chelating Growth Factors from Mycobacteria. Bacteriol Rev 34: 99-125 (1970)

8. Tunakawa M, Chang L, Mamber SW, Bursuker I, Hugill R. (Bristol-Myers Squibb Company) Antitumor antibiotic BMS-199687. U.S. 5,811,440, September 22 (1998)

9. Wagastuma T, Kizuka M, Kurakata S, Shiozawa H,
Nakajima M, Furukawa H. (Sankyo Co., Ltd.) Japan Pat. 00,344,768, December 12 (2000)

10. Tsuda M, Yamakawa M, Oka S, Tanaka Y, Hoshino Y, Mikami Y, Sato A, Fujiwara H, Ohizumi Y, Kobayashi J. Brasilibactin A, a cytotoxic compound from actinomycete Nocardia brasiliensis. J Nat Prod 68: 462-464 (2005) 